\title{
A geostatistical model for combined analysis of point-level and area-level data using INLA and SPDE
}

\author{
Paula Moraga ${ }^{\mathrm{a}, *}$, Susanna M. Cramb ${ }^{\mathrm{a}, \mathrm{b}}$, Kerrie L. Mengersen ${ }^{\mathrm{a}, \mathrm{c}}$, Marcello \\ Pagano $^{\mathrm{d}}$ \\ ${ }^{a}$ ARC Centre of Excellence for Mathematical $\mathcal{G}$ Statistical Frontiers, Queensland University \\ of Technology (QUT), Brisbane, Australia \\ ${ }^{b}$ Cancer Council Queensland, Brisbane, Australia \\ ${ }^{c}$ Cooperative Research Centre for Spatial Information, Australia \\ ${ }^{d}$ Department of Biostatistics, Harvard T.H. Chan School of Public Health, Boston, United \\ States
}

\begin{abstract}
In this paper a Bayesian geostatistical model is presented for fusion of data obtained at point and areal resolutions. The model is fitted using the INLA and SPDE approaches. In the SPDE approach, a continuously indexed Gaussian random field is represented as a discretely indexed Gaussian Markov random field (GMRF) by means of a finite basis function defined on a triangulation of the region of study. In order to allow the combination of point and areal data, a new projection matrix for mapping the GMRF from the observation locations to the triangulation nodes is proposed which takes into account the types of data to be combined. The performance of the model is examined and compared with the performance of the method RAMPS via simulation when it is fitted to i) point, ii) areal, and iii) point and areal data to predict several simulated surfaces that can appear in real settings. The model is applied to predict the concentration of fine particulate matter $\left(\mathrm{PM}_{2.5}\right)$, in Los Angeles and Ventura counties, United States, during 2011.
\end{abstract}

Keywords: Misalignment, Data fusion, Model-based geostatistics, INLA, SPDE

*Paula Moraga, School of Mathematical Sciences, Queensland University of Technology, GPO Box 2434, Brisbane, QLD 4001, Australia. E-mail: paula.moragaserrano@qut.edu.au

Preprint submitted to Spatial Statistics

April 14, 2017 


\section{Introduction}

Spatial and spatio-temporal data arise in a wide range of scientific disciplines, including the environmental, epidemiological, geographical and ecological fields 1]. Data are typically observed either at points in space (point data), or over 5 areal units such as counties or postal codes (areal data). Examples include air pollution measurements taken at a set of ambient stations, temperature and precipitation measurements from weather stations, and population sizes from census tracts. In epidemiology, point data arise when the locations at which cases of disease occur are available, and areal data are often reported when point data are aggregated over geographical subregions of the region of study due to ethical concerns over data use and patient confidentiality [2].

Spatially misaligned data are becoming increasingly common due to advances in both data collection and management, as well as to the ability to merge data from large databases such as disease registries. When information is available from multiple sources on different scales, data may be fused to examine just one variable, such as disease counts recorded in different administrative units. Here the aim is interpolation [3]. Alternatively, we might wish to relate one variable to other variables that are available at different spatial resolutions and alignments. An example is determining whether the risk of an adverse outcome provided at zip level is related to exposure to an environmental pollutant measured at a network of stations, after adjusting for population at risk and other county level demographic information. Here the aim is regression [3].

In this paper we will focus on the data fusion problem which seeks to learn about a particular variable by combining data that are available at different 25 spatial scales. Others have previously developed Bayesian models enabling fusion of data obtained at areal and point-referenced resolutions via the use of latent point-level processes 4], hierarchical downscaling [5], modelling data conditional on the resolution [6], and the use of algorithms such as the reparameterized and marginalized posterior sampling (RAMPS) [7.

The previous approaches use Bayesian predictive inference implemented via 
Markov chain Monte Carlo (MCMC) based methods. These methods have made a great impact on statistical practice by making Bayesian inference tractable for complex models but they also present a wide range of problems in terms of convergence and computational time [8]. In this paper we propose general and flexible hierarchical Bayesian models to analyze spatially misaligned data. In order to fit the models, we resort to the Integrated Nested Laplace approximation (INLA) 9] and the Stochastic Partial Differential Equation (SPDE) [10] approaches which are a computationally effective alternative to MCMC for Bayesian inference. In order to allow the combination of data at different spatial resolutions, we propose a new projection matrix for mapping the GMRF in the SPDE method which takes into account how the different types of data are collected. This new approach is fast and flexible.

The outline of the paper is as follows. First, we present flexible models for handling spatial misaligned data in fusion problems. Then, we briefly introduce

45 the INLA and SPDE approaches for Bayesian inference, and present the projection matrix that allows the combination of point and areal data. In Section 3. a simulation study is carried out to compare the performance of the model when estimating several simulated surfaces using point, areal, and point and areal data combined. Then, in Section 4 we evaluate the model in comparison to the RAMPS alternative method for data fusion by applying the methods to several simulated data scenarios. In Section 5 we present an application of the model to real data showing spatial misalignment. In this application, we obtain the spatial distribution of fine particulate matter $\left(\mathrm{PM}_{2.5}\right)$, in Los Angeles and Ventura counties, United States, during 2011. Finally, the conclusions are presented.

\section{Models and Inference}

\subsection{Models}

The models proposed assume that there is a spatially continuous variable underlying all observations that can be modeled using a Gaussian random field 
process. This process is denoted by $S=\left\{S(\mathbf{x}): \mathbf{x} \in D \subset \mathbb{R}^{2}\right\}$, has mean function $E[S(\mathbf{x})]=0$ and stationary covariance function $\operatorname{Cov}\left(S(\mathbf{x}), S\left(\mathbf{x}^{\prime}\right)\right)=$ $\Sigma\left(\mathbf{x}-\mathbf{x}^{\prime}\right)$. Conditionally on $S$, point data $Y_{i}$ observed at a finite set of sites, say $\mathbf{x}_{i} \in D, i=1,2, \ldots, I$, are mutually independent with

$$
Y_{i} \mid S\left(\mathbf{x}_{i}\right) \sim N\left(\mu\left(\mathbf{x}_{i}\right)+S\left(\mathbf{x}_{i}\right), \tau^{2}\right),
$$

where $\mu\left(\mathbf{x}_{i}\right)$ represents the large scale structure. Areal data observations arise as block averages in blocks $B_{j} \subset D, j=1,2, \ldots, J$,

$$
Y\left(B_{j}\right)=\left|B_{j}\right|^{-1} \int_{B_{j}}(\mu(\mathbf{x})+S(\mathbf{x})) d \mathbf{x},\left|B_{j}\right|>0,
$$

where $\left|B_{j}\right|=\int_{B_{j}} 1 d \mathbf{x}$ denotes the area of $B_{j}$.

These models can also accommodate explanatory covariates by including them in the large scale part of the model. Moreover, the models can also be extended to include random effects that can deal with other sources of variability.

\subsection{Inference}

We fit the models by using the INLA [11, 9] and SPDE approaches [10] which can be easily applied using the R package R-INLA [12]. INLA uses a combination of analytical approximation and numerical integration to do approximate Bayesian inference in latent Gaussian models which includes a large class of models ranging from generalized linear mixed to spatial and spatio-temporal models.

The combination of INLA and SPDE permits analysis of point-level data. In the SPDE approach, the continuously indexed Gaussian field $S$ is represented as a discretely indexed Gaussian Markov random field (GMRF) by means of a finite basis function defined on a triangulation of the region of study. Specifically,

$$
S(\mathbf{x})=\sum_{g=1}^{G} \psi_{g}(\mathbf{x}) S_{g},
$$

where, $\psi_{g}(\cdot)$ denotes piecewise polynomial basis functions on each triangle, $\left\{S_{g}\right\}$ are zero-mean Gaussian distributed weights, and $G$ are the number of vertices in the triangulation. 
In the SPDE approach, the covariance function of the Gaussian field $S$ is required to belong to the Matérn family which represents a very flexible class of covariance functions that appears naturally in many scientific fields [13. Specifically, for locations $\mathbf{x}_{i}$ and $\mathbf{x}_{j} \in \mathbb{R}^{2}$, the Matérn covariance function is defined as

$$
\operatorname{Cov}\left(S\left(\mathbf{x}_{i}\right), S\left(\mathbf{x}_{j}\right)\right)=\frac{\sigma^{2}}{2^{\nu-1} \Gamma(\nu)}\left(\kappa\left\|\mathbf{x}_{i}-\mathbf{x}_{j}\right\|\right)^{\nu} K_{\nu}\left(\kappa\left\|\mathbf{x}_{i}-\mathbf{x}_{j}\right\|\right)
$$

Here, $K_{\nu}$ is the modified Bessel function of second kind and order $\nu>0$. The integer value of $\nu$ determines the mean square differentiability of the process and it is usually fixed since it is poorly identified in applications. $\sigma^{2}$ denotes

75 the variance and $\kappa>0$ is related to the range $\rho$, the distance at which the spatial correlation is close to 0.1 [14].

\subsection{Approximation of integrals}

In practice, the integrals appearing in the models proposed may not be available in a closed-form. In our approach, we will approximate them using the representation of the continuous Gaussian random field as a GMRF provided by the SPDE approach. Thus,

$$
\int_{B_{j}} S(\mathbf{x}) d \mathbf{x} \approx \sum_{g=1}^{G} A_{j g} S_{g},
$$

where $G$ is the number of vertices in the triangulation, $\left\{S_{g}\right\}$ are zero-mean Gaussian distributed weights, and $A$ is a $J \times G$ sparse matrix that maps the

so GMRF from the $J$ observation locations to the $G$ triangulation nodes.

The matrix $A$ specified in the SPDE approach is designed to deal with pointreferenced data. To adapt this approach to our problem, we need to make some modifications that allow accommodation of both point and areal data. Specifically, for the construction of $A$, we need to differentiate between point 85 and areal observations. If we consider observations taken at point locations in the study region, the projection matrix $A$ can be chosen in the same way as in the SPDE approach. Thus, the row $i$ in $A$ corresponding to an observation at point $\mathbf{x}_{i}$, will possibly have three non-zero values at the columns that represent the 
vertices of the triangle that contains the point. If $\mathbf{x}_{i}$ is within the triangle, these that have been obtained at several configurations of points and areas in the region of study. First, we generate several spatial surfaces that may reproduce some of the situations that can appear in real settings. Then, for each of the surfaces, the model is fitted using point and areal measurements of the surfaces 115 the simulated situations are evaluated. The rest of this section describes the geographic region and the data configurations we decide to use throughout the 
simulations, the models used to generate the spatial surfaces, the models fitted, and the results of the simulation study.

\subsection{Simulated data}

We are interested in testing the method in a range of situations that can appear in real settings. To do so, we decide to simulate several spatial surfaces of phenomena that can have continuous values in $\mathbb{R}$. These data may represent, for example, concentration levels of some air pollutant, and may be measured directly at points where monitoring stations are located, or may be obtained at cells of a regular grid produced by numerical models. In our simulation, we consider the unit square as the study region and take observations at different configurations of randomly generated points and regular grids and vertical bands over the region of study.

The different data scenarios are created by varying the number of points and areas in the data sets to be combined. Specifically, data sets of 10, 15, 30, 60 and 100 points are combined with data sets of 4, 16 and 100 squared areas, or 2, 4 and 10 vertical areas. We also create scenarios with no point data and scenarios with no areal data. Examples of such configurations are shown in Figure 1.

We construct four surfaces for simulated data. For locations $\mathbf{x}_{i}$ in the unit square, observations $Y_{i}$ in $\mathbb{R}$ are simulated as follows:

$$
Y_{i}=\boldsymbol{z}_{i} \boldsymbol{\beta}+S\left(\mathbf{x}_{i}\right), i=1, \ldots, n,
$$

where $\boldsymbol{z}_{i}=\left(1, z_{i}\right)$ denotes the vector of the intercept and covariates, $\boldsymbol{\beta}=$ $\left(\beta_{0}, \beta_{c}\right)^{\prime}$ is the coefficient vector, and $S$ is a zero-mean Gaussian field with Matérn covariance function with variance $\sigma^{2}$ and range $\rho$. In the simulations, we set the intercept $\beta_{0}=0, \sigma^{2}$ equal to 4 or 1 and $\rho$ equal to 0.7 or 0.1 . Moreover, we use as a covariate a geographic trend with $\beta_{c}=2$. The trend covariate is calculated as $\left(x_{i}^{2}-\overline{x^{2}}\right)$, where $x_{i}^{2}$ is the second coordinate of location $\mathbf{x}_{i}$, and $\overline{x^{2}}$ is the mean of the second coordinate over the study region. In this way, we are generating a surface where the values increase from south to north. This could 

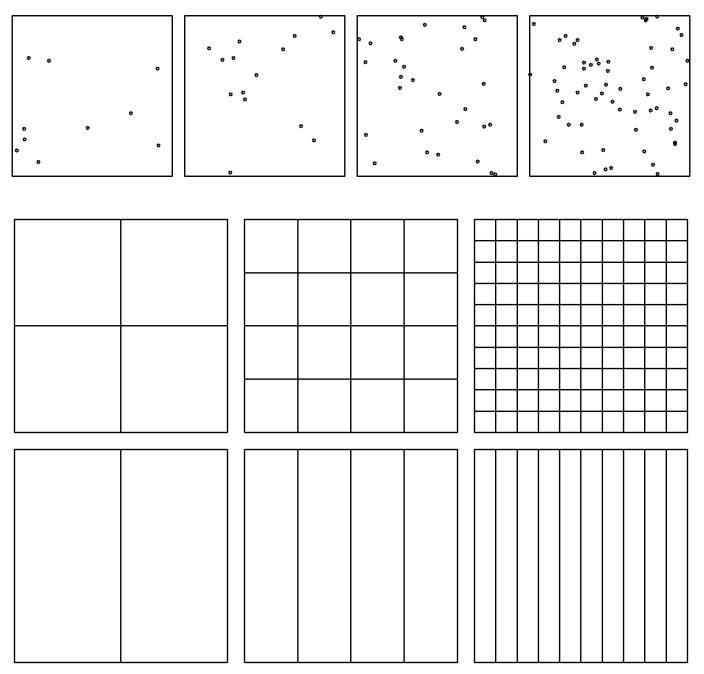

Figure 1: Examples of point and areal configurations used in the simulation study.

represent a surface that reflects changes in temperature or other environmental covariates that are related with latitude. The values of the parameters for each of the simulated surfaces are presented in Table 1. Examples of the simulated surfaces are shown in Figures 2 to 5.

\begin{tabular}{ccccc} 
& covariate & $\beta_{c}$ & $\sigma^{2}$ & $\rho$ \\
\hline US1 & - & - & 4 & 0.7 \\
US2 & - & - & 1 & 0.1 \\
US3 & trend & 2 & 4 & 0.7 \\
US4 & trend & 2 & 1 & 0.1
\end{tabular}

Table 1: Parameters of the models used to generate the surfaces in the simulation study.

\subsection{Fitted models}

Let $Y_{i}, i=1, \ldots, n+m$, denote the simulated observations at points $\mathbf{x}_{i}$, $i=1, \ldots, n$, and areas $B_{i}, i=n+1, \ldots, n+m$. The fitted models assume a Normal likelihood with mean $\mu_{i}$ for the first level, and the following structure 
for $\mu_{i}$ in the second level:

$$
\begin{gathered}
\mu_{i}=\boldsymbol{z}_{i} \boldsymbol{\beta}+S\left(\mathbf{x}_{i}\right), i=1, \ldots, n \\
\mu_{i}=\boldsymbol{z}_{i} \boldsymbol{\beta}+\left|B_{i}\right|^{-1} \int_{B_{i}} S(\mathbf{x}) d \mathbf{x}, i=n+1, \ldots, n+m .
\end{gathered}
$$

Here, $\boldsymbol{z}_{i}=\left(1, z_{i}\right)$ denotes the vector of the intercept and the covariate, Matérn covariance function with parameters $\sigma^{2}$ and $\rho$. If the data are simulated without a covariate, the fitted models do not incorporate the covariate term. If they are simulated using a covariate, the models incorporate the effect of the same covariate. parameter $\nu$ is set fixed to 1 in the Matérn function implying a continuous domain Markov field. We assign a flat improper prior to the intercept $\beta_{0}$, and a zero-mean Gaussian distribution with precision equal to 0.001 for the effect of the covariate. Finally, $S \sim N\left(0, Q^{-1}\right)$ where $Q$ is a sparse precision matrix depending on hyperparameters $\kappa$ and $\sigma^{2}$.

\subsection{Results}

For each simulated pattern, we generate point and areal data and predict the simulated surface applying the model to i) point, ii) areal, and iii) point and areal data combined. We generate 100 surfaces from each simulated scenario to have stable results. The merits of the model in each situation are assessed using the mean squared errors (MSE) of the predictions. The MSE for each simulated data set is calculated as

$$
\operatorname{MSE}=\left(\frac{1}{R} \sum_{\mathbf{x} \in R}(u(\mathbf{x})-\hat{u}(\mathbf{x}))^{2}\right)^{1 / 2},
$$

where $R$ denotes the number of locations in the study region, $u(\mathbf{x})$ is the value of the simulated surface at location $\mathbf{x}$, and $\hat{u}(\mathbf{x})$ is the prediction of $u(\mathbf{x})$. Figures 2 to 5 show the MSEs for each of the scenarios and combinations of data averaged 
The results show that the MSE depends on the simulated surfaces and also on the types of data used to fit the model. We observe that when the model is applied using data obtained in just areas or points, the MSE decreases as the number of areas or points increases. There are some situations, however,

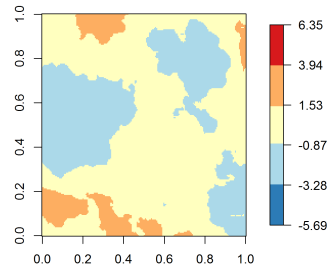

Areal analysis Point analysis Joint analysis
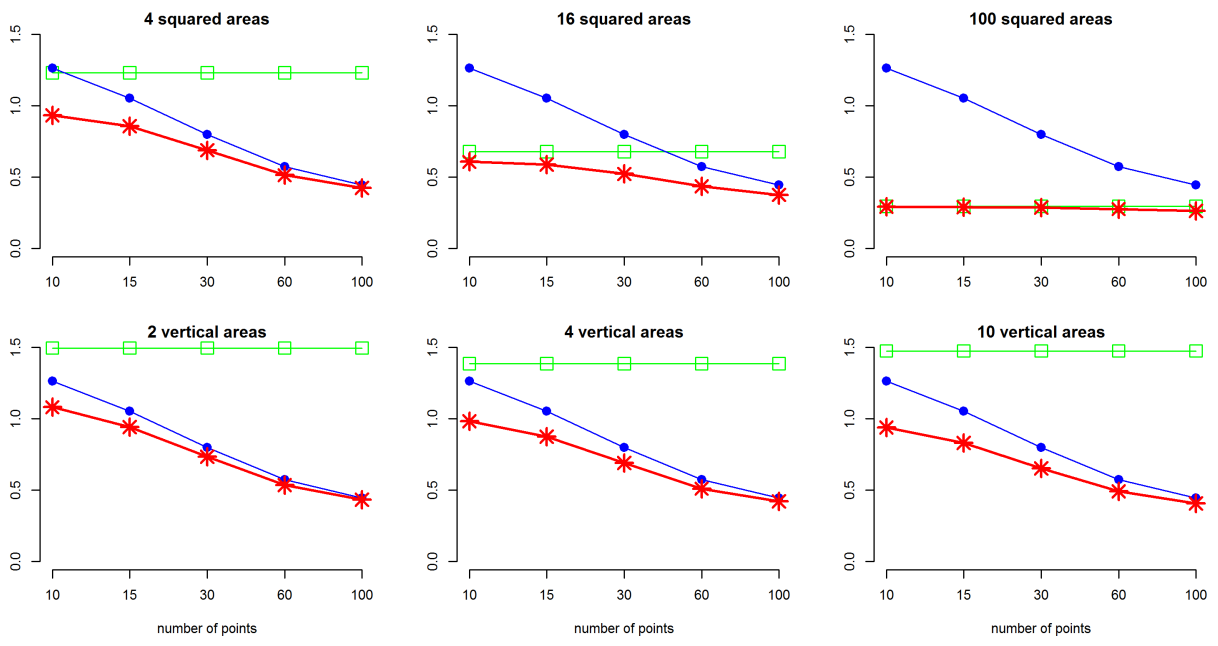

Figure 2: Scenario US1 results. First column: one of the 100 simulated surfaces. Second to fourth columns: MSEs of the predictions obtained for the simulated surfaces US1 averaged over 100 replications by type of analysis. 


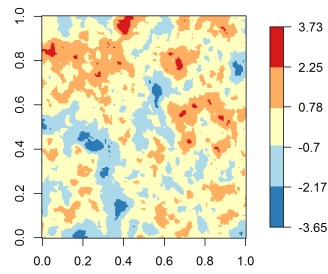

Areal analysis Point analysis Joint analysis
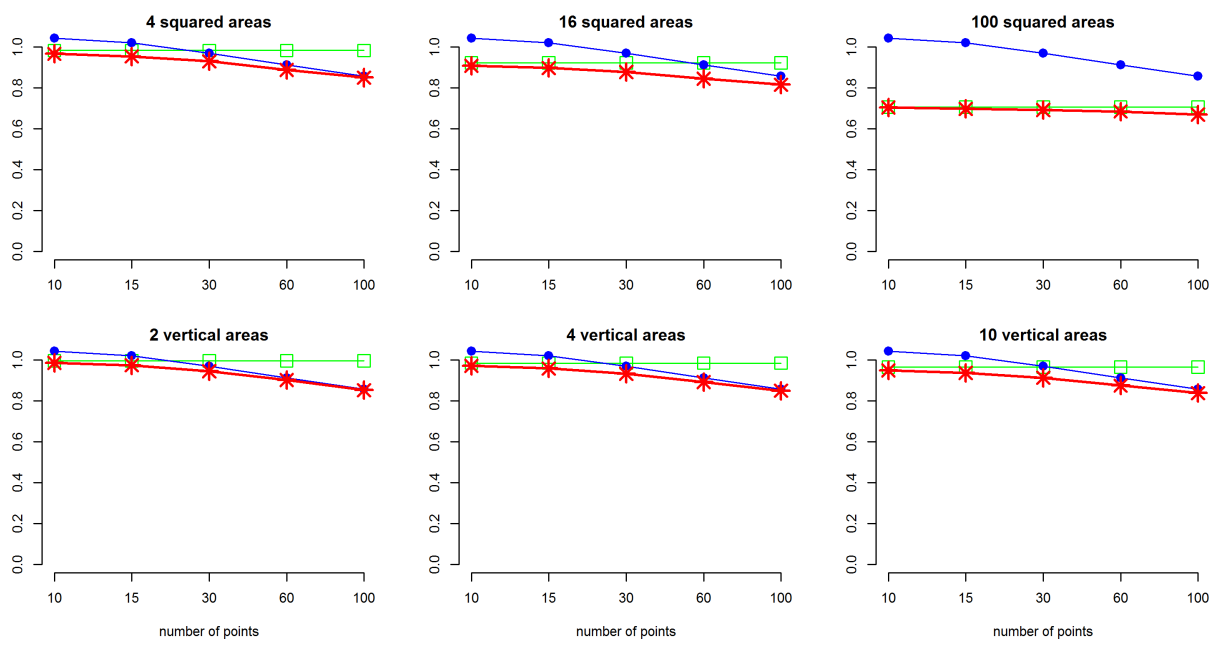

Figure 3: Scenario US2 results. First column: one of the 100 simulated surfaces. Second to fourth columns: MSEs of the predictions obtained for the simulated surfaces US2 averaged over 100 replications by type of analysis.

\section{Performance evaluation in comparison with RAMPS}

In this section, we compare the method presented with another existing method for data fusion. Specifically, we present a performance evaluation of our method in comparison with the reparameterized and marginalized posterior sampling (RAMPS) algorithm for complex Bayesian geostatistical models. We chose RAMPS for comparison because of its flexibility and the availability of an $\mathrm{R}$ package called ramps that implements all of its capabilities [15. RAMPS enables joint modeling of areal and point data arising from the same underlying spatial process, and allows accommodation of non-spatial correlation and variance heterogeneity as well as spatial and/or temporal correlation. Specifically, an observation vector $Y$ which may contain both point and areal data is modeled as follows:

$$
\begin{gathered}
Y=X \beta+W \gamma+K Z+\epsilon, \\
\gamma \sim N\left(0, \Sigma_{\gamma}\right), Z \sim N\left(0, \Sigma_{Z}\right), \epsilon \sim N\left(0, \Sigma_{\epsilon}\right),
\end{gathered}
$$




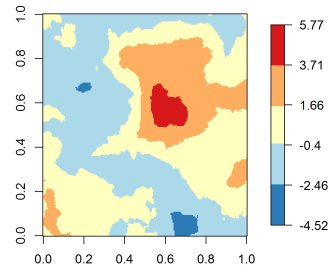

Areal analysis Point analysis Joint analysis
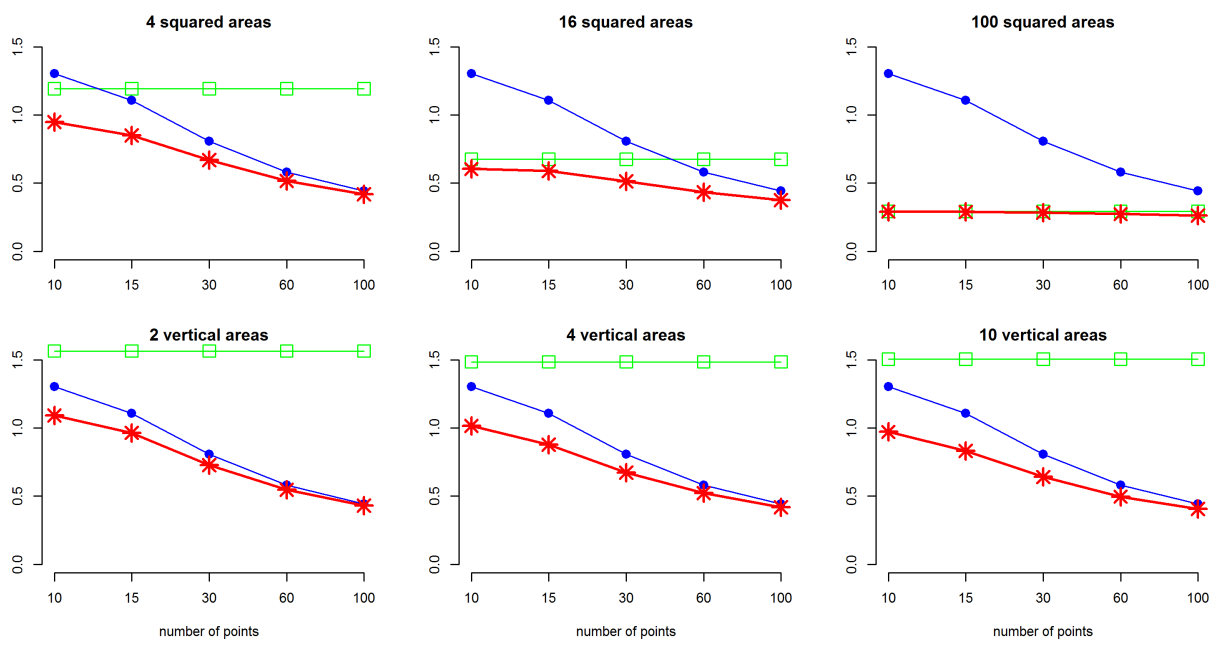

Figure 4: Scenario US3 results. First column: one of the 100 simulated surfaces. Second to fourth columns: MSEs of the predictions obtained for the simulated surfaces US3 averaged over 100 replications by type of analysis.

where $\beta$ is a vector of regression coefficients, $\gamma$ is a vector of non-spatial random effects, $Z$ is an vector of spatial random effects, $\epsilon$ is a vector of measurements errors, and the matrices $X, W$, and $K$ are design matrices for fixed effects, non-spatial random effects, and spatial random effects, respectively. The model is fitted using an algorithm that involves reparameterizing the variance parameters, reformulating the means structure, marginalizing the joint posterior distribution, and applying the slice sampling MCMC method based on simplexes.

Here, we simulate four surfaces with different characteristics and obtain point and areal observations. Then, our method and RAMPS are applied to predict the simulated surfaces using all the observations combined. Finally, the performance of the methods is evaluated by means of the MSE, the parameter estimates and the run time.

The four spatial surfaces are generated on $[0,1] \times[0,1]$ using a Gaussian model with a Matérn covariance structure with variance $\sigma^{2}$, range $\rho$ and overall mean $\beta_{0}=0$. For surfaces S1 and S3, we set $\sigma^{2}=4$ and $\rho=0.7$, in surfaces S2 and S4, $\sigma^{2}=1$ and $\rho=0.1$. Moreover, in surfaces S3 and S4 we use a 


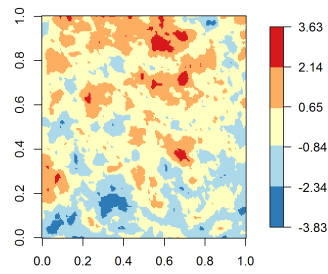

Areal analysis Point analysis Joint analysis
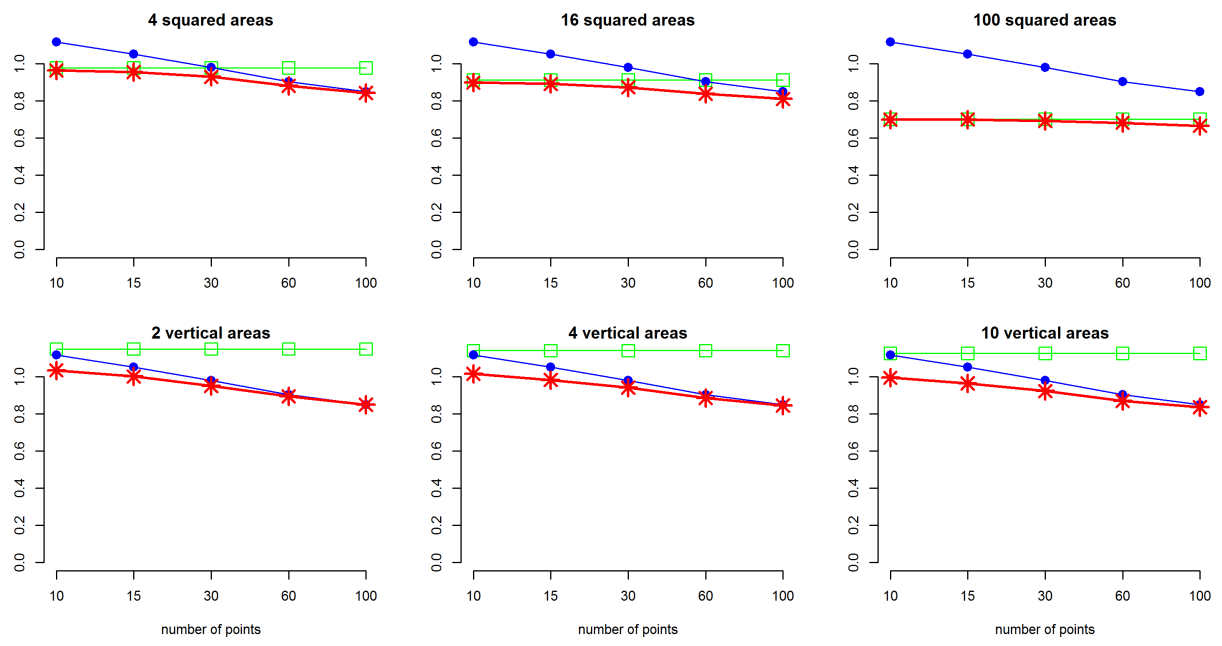

Figure 5: Scenario US4 results. First column: one of the 100 simulated surfaces. Second to fourth columns: MSEs of the predictions obtained for the simulated surfaces US4 averaged over 100 replications by type of analysis.

geographic trend covariate calculated as $\left(x_{i}^{2}-\overline{x^{2}}\right)$ and coefficient $\beta_{c}=2$. The observations to be combined are obtained as the point values corresponding to 100 randomly generated locations and the average values in the cells of a $4 \times 4$ regular grid. The surfaces generated and the areal and point observations for each scenario are shown in Figure 6.

We apply our method and RAMPS to predict the simulated surfaces. Let $Y_{i}, i=1, \ldots, n+m$, denote the simulated observations at points $\mathbf{x}_{i}, i=1, \ldots, n$, and areas $B_{i}, i=n+1, \ldots, n+m$. The model fitted assumes a Normal likelihood with mean $\mu_{i}$ expressed as

$$
\begin{gathered}
\mu_{i}=\boldsymbol{z}_{i} \boldsymbol{\beta}+S\left(\mathbf{x}_{i}\right), i=1, \ldots, n, \\
\mu_{i}=\boldsymbol{z}_{i} \boldsymbol{\beta}+\left|B_{i}\right|^{-1} \int_{B_{i}} S(\mathbf{x}) d \mathbf{x}, i=n+1, \ldots, n+m .
\end{gathered}
$$

Here, $\boldsymbol{z}_{i}=\left(1, z_{i}\right)$ is the vector of the intercept and the covariate, $\boldsymbol{\beta}=\left(\beta_{0}, \beta_{c}\right)^{\prime}$ is the coefficient vector, and $S$ is a zero-mean Gaussian field with Matérn covariance function with parameters $\sigma^{2}$ and $\rho$. This model is fitted to the data simulated using a covariate. If the data are simulated without covariate, the 
fitted model does not incorporate the covariate term. Due to the large amount of time needed to fit the model using RAMPS, prediction is just done at 231 uniformly distributed locations.

The priors used when applying our method are the same as the ones employed in the simulation study in Section 3. When applying RAMPS, flat priors are used on the intercept $\beta_{0}$ and the covariate coefficient $\beta_{c}$. An inverse gamma prior with shape and scale parameters set to 0.01 is used for $\sigma^{2}$, and an uniform prior on $(0,2)$ is used for the range $\rho$. Using RAMPS, convergence is achieved running a MCMC chain of 30,000 iterations and using a burn-in of 1,000 and a thinning rate of 30 iterations for each of the surfaces. Posterior means and 95\% CIs are calculated with the remaining 966 iterations. We note that the use of different priors may result in different estimates and run times. However, showing results from even only these priors reveals the main differences in performance between our method and RAMPS.

For each of the simulated surfaces and methods, we calculate the MSE, the posterior means and $95 \%$ CIs for the model parameters, and the run times. These values are shown in Table 2. We observe that lower MSEs are obtained with our method than with RAMPS in all surfaces except for surface S2, where ours is 0.02 higher ( 0.89 with our method and 0.87 with RAMPS). The highest difference in MSE is obtained when the methods are applied to predict surface S3 which is simulated using a geographic trend as a covariate, $\sigma^{2}=4$ and $\rho=0.7$. In S3, the MSE obtained with our method is equal to 0.46 compared to 1.75 with RAMPS. We see that neither our method nor RAMPS accurately recover the true values of the parameters used in the simulations, both methods yield $95 \%$ CIs that contain the true values for most of the parameters. We also see that in the simulated surfaces S1 and S3, that is, when $\sigma^{2}=4$ and $\rho=0.7$, the upper limits of the $95 \%$ CIs for $\sigma^{2}$ obtained with RAMPS are very high. With our method, however, narrower $95 \%$ CIs for $\sigma^{2}$ are obtained. Finally, we 235 note that RAMPS needs longer run times than our method. Specifically, for all surfaces RAMPS takes more than three hours whereas our method finishes in less than one minute. 


\section{Application to air pollution data}

The methodology proposed provides a valuable tool in a wide range of re-

240 tribution of a common air pollutant: fine particulate matter $\left(\mathrm{PM}_{2.5}\right)$ in Los Angeles and Ventura counties, United States, during 2011. We fit a spatial model combining information for the variable of interest from point and areal resolutions. We also model point and areal data separately to assess the differ-

pollutants [17] We have used the mean of the daily measurements recorded in year 2011 in each of the monitoring stations. The satellite-derived estimates represent three-year mean grids (2010-2012) of $\mathrm{PM}_{2.5}$ concentrations derived from a combination of MODIS (Moderate Resolution Imaging Spectroradiometer), [18, 19]. The raster grid has a grid cell resolution of 6 arc-minutes (0.1 degree 
or approximately $10 \mathrm{~km}$ at the equator). Figure 7 shows the concentration values in each of the monitoring stations and in the raster grid.

The model used to predict $\mathrm{PM}_{2.5}$ values in the study region is specified as follows. The $\mathrm{PM}_{2.5}$ concentration, $Y_{i}$, at each of the locations of the monitoring stations, $\mathbf{x}_{i}, i=1, \ldots, n$, and cells of the raster grid, $B_{i}, i=n+1, \ldots, n+m$, are modeled as Gaussian observations with mean $\mu_{i}$ :

$$
\begin{gathered}
Y_{i} \sim \operatorname{Normal}\left(\mu_{i}, \sigma^{2}\right), i=1, \ldots, n, n+1, \ldots, n+m, \\
\mu_{i}=\beta_{0}+S\left(\mathbf{x}_{i}\right), i=1, \ldots, n, \\
\mu_{i}=\beta_{0}+\left|B_{i}\right|^{-1} \int_{B_{i}} S(\mathbf{x}) d \mathbf{x}, i=n+1, \ldots, n+m,
\end{gathered}
$$
covariance function with parameters $\sigma^{2}$ and $\rho$. We fit the model three times using areal, point and areal and point data. The model is fitted using the same priors as the ones employed in the simulation study in Section 3 .

Although estimates differed by analysis the 95\% CIs overlapped (Table 3). The most accurate predictions (tightest CIs) for model parameters were generally for the areal model, then our model, while using only the point data resulted in large uncertainty around the estimates.

Maps of the predictions obtained and the 95\% CI showing the range of plausible values for each location are shown in Figure 8. Although all maps show a predicted $\mathrm{PM}_{2.5}$ higher in the south, there are some differences depending on the analysis used. For example, with the joint analysis the predicted $\mathrm{PM}_{2.5}$ is higher close to the city of Los Angeles than the predicted $\mathrm{PM}_{2.5}$ obtained with the areal analysis. Also, by using both areal and point data, we are able to obtain more accurate predictions in the south where there is both point and areal information than the ones obtained using just one type of data.

\section{Discussion}

In this paper we have presented a joint Bayesian model to combine point and areal data. The model assumes that underlying all observations there is a 
spatially continuous variable that can be modeled using a Gaussian random field process. INLA and SPDE approaches were used to fit the data and represent the continuously indexed Gaussian random field as a discretely indexed GMRF by means of a basis function representation defined on a triangulation of the region of interest. In order to allow the combination of point and areal data, we proposed a new projection matrix for mapping the GMRF from the observation locations to the triangulation nodes which takes into account the types of data.

The results show that the goodness of fit depends on the simulated surfaces and also the types of data used to fit the model. In most situations we observe that the combination of point and areal data provides better predictions than if the method is applied to just one type of data, and this was consistent over both simulated and real data. Our method was also demonstrably superior to RAMPS by obtaining better predictions in much shorter run times on simulated data.

Real data are messy, especially when attempting to use multiple sources of information. Our method performed well when applied to monitored air pollution data. When predicting the concentration of $\mathrm{PM}_{2.5}$ in Los Angeles and Ventura counties during 2011, the point analysis gave markedly different results to the areal analysis (Figure 8p. In part, this may be due to differences in time periods, as well as the relative lack of monitoring stations in the north. Combining these estimates using our method enabled more accurate predictions of the concentration of $\mathrm{PM}_{2.5}$, particularly in the south.

A limitation of the method proposed is that it is only applicable to Gaussian data. Unfortunately, this does not include many important settings such as disease mapping problems where data are typically modelled using Poisson or Binomial distributions and non-identity link functions.

Models based on aggregated data contain the potential for ecological fallacy which occurs when estimated associations obtained from analyses of variables measured at an aggregated level lead to conclusions different from analyses based on the same variables measured at the individual level. The resulting bias, called ecological bias, is comprised of two effects: the aggregation bias due 
to the grouping of individuals, and the specification bias due to the differential distribution of confounding variables created by grouping [20, [21]. In many situations, however, it is difficult to obtain sufficient point data to obtain conclusions and we should make the most of the information from the available data regardless of their spatial resolution. For example, [22] show how the com325 bination of disease data from different sources can improve inferences from that using a single data set, and demonstrate that analyses combining related data at both the individual and aggregate level can reduce ecological bias and add precision.

There are many situations where data are very hard to obtain and especially in these cases it is very important to optimize the use of all available information. The method proposed enables obtaining better predictions by combining data obtained at different resolutions. However, we should be aware that bias could arise if we combine data that are not completely comparable such as data collected about different populations or at different times. In such situations we may decide to use just one of the data sets or alternatively to adjust for bias in the model.

A major advantage of the method presented is that the Bayesian framework used could be easily extended to adequately model many problems of interest. For example, the model may be extended to accommodate spatio-temporal data as follows. Let us consider a spatio-temporal Gaussian process $S=\{S(\mathbf{x}, t)$ : $\left.\mathbf{x} \in D \subset \mathbb{R}^{2}, t \in T \subset \mathbb{R}\right\}$ with $E[S(\mathbf{x}, t)]=0$ and stationary covariance function $\operatorname{Cov}\left(S(\mathbf{x}, t), S\left(\mathbf{x}^{\prime}, t^{\prime}\right)\right)=\Sigma\left(\mathbf{x}-\mathbf{x}^{\prime}, t-t^{\prime}\right)$. Data observed at locations $\mathbf{x}_{i}, i=1, \ldots, I$, and times $t_{k}, k=1, \ldots, K$ may be modeled as

$$
Y_{i, k} \mid S\left(\mathbf{x}_{i}, t_{k}\right) \sim N\left(\mu\left(\mathbf{x}_{i}, t_{k}\right)+S\left(\mathbf{x}_{i}, t_{k}\right), \tau^{2}\right)
$$

Then, observations in areas $B_{j} \subset D, j=1,2, \ldots, J$, and periods of time $\tau_{l} \in T$, $l=1, \ldots, L$, are expressed averaging the process in space and also in time,

$$
Y\left(B_{j}, \tau_{l}\right)=\left|B_{j}\right|^{-1}\left|\tau_{l}\right|^{-1} \int_{B_{j}} \int_{\tau_{l}}(\mu(\mathbf{x}, t)+S(\mathbf{x}, t)) d \mathbf{x} d t,\left|B_{j}\right|>0,\left|\tau_{l}\right|>0
$$

Also, it is possible to include covariates, and handling and representing different 
sources of uncertainty, including sampling error, measurement error, as well as prediction errors at unsampled locations. Another advantage of the method is that by using the approximate methods INLA and SPDE, we are able to obtain results quickly and avoid assessing the convergence and mixing properties of the chains generated by using MCMC-based methods. In addition, since this method is less computationally intensive we are able to deal with large data sets.

The combination of point-level and area-level referenced data is an important and not yet completely resolved methodological issue within the general area of spatial statistics. We think that the approach presented may be a helpful advance in this area by providing a useful tool that is applicable in a wide range of situations where information at different spatial resolutions is combined.

\section{Acknowledgements}

PM would like to acknowledge the financial support for this research from NIH/NIAID grant R01AI097015-01A1 and ARC Australian Laureate Fellowship FL150100150.

\section{References}

355 [1] N. Cressie, Statistics for spatial data, Wiley, New York, 1993.

[2] A. B. Lawson, Bayesian point event modeling in spatial and environmental epidemiology, Statistical Methods in Medical Research 21 (2012) 509-529.

[3] S. Banerjee, B. P. Carlin, A. E. Gelfand, Hierarchical modeling and analysis for spatial data, Second Edition, Chapman and Hall/CRC, Boca Raton, 2014.

[4] M. Fuentes, A. E. Raftery, Model evaluation and spatial interpolation by bayesian combination of observations with outputs from numerical models, Biometrics 66 (2005) 36-45. 
[5] V. J. Berrocal, A. E. Gelfand, D. M. Holland, A spatio-temporal downscaler for outputs from numerical models, J. Agric. Biol. Environ. Stat. 15 (2010) 176-197.

[6] C. K. Wikle, L. M. Berliner, Combining information across spatial scales, Technometrics 47 (1) (2005) 80-91.

[7] M. K. Cowles, J. Yan, B. Smith, Reparameterized and marginalized posterior and predictive sampling for complex bayesian geostatistical models, Tech. rep., The University of Iowa, Department of Statistics and Actuarial Science (2007).

URL http://www.stat.uiowa.edu/techrep/

[8] B. M. Taylor, P. J. Diggle, INLA or MCMC? A tutorial and comparative evaluation for spatial prediction in log-Gaussian Cox processes, Journal of Statistical Computation and Simulation 84 (10) (2014) 2266-2284.

[9] H. Rue, S. Martino, N. Chopin, Approximate Bayesian inference for latent Gaussian models by using integrated nested Laplace approximations, Journal of the Royal Statistical Society, Series B 71 (2) (2009) 319-392.

[10] F. Lindgren, H. Rue, J. Lindström, An explicit link between gaussian fields and gaussian markov random fields: the stochastic partial differential equation approach, Journal of the Royal Statistical Society, Series B 73 (4) (2011) 423-498.

[11] H. Rue, S. Martino, Approximate Bayesian inference for hierarchical Gaussian Markov random fields models, Journal of Statistical Planning and Inference 137 (2007) 3177-3192.

[12] H. Rue, S. Martino, F. Lindgren, D. Simpson, A. Riebler, R-inla: Approximate bayesian inference using integrated nested laplace approximations, http://www.r-inla.org/ (2013).

390

[13] P. Guttorp, T. Gneiting, Studies in the history of probability and statistics xlix on the matérn correlation family, Biometrika 93 (4) (2006) 989-995. 
[14] M. Cameletti, F. Lindgren, D. Simpson, H. Rue, Spatio-temporal modeling of particulate matter concentration through the spde approach, AStA Advances in Statistical Analysis.

[15] B. J. Smith, J. Yan, M. K. Cowles, Unified geostatistical modeling for data fusion and spatial heteroskedasticity with r package ramps, Journal of Statistical Software 25 (10) (2008) 1-21.

[16] United States Environmental Protection Agency (EPA), Particulate Matter (PM) Pollution, https://epa.gov/pm-pollution (2015).

[17] United States Environmental Protection Agency (EPA), Air Qual-

口 ity Data Mart, https://aqs.epa.gov/aqsweb/documents/data_mart_ welcome.html (2015).

[18] A. van Donkelaar, R. V. Martin, M. Brauer, B. L. Boys, Global annual pm2.5 grids from modis, misr and seawifs aerosol optical depth (aod), 1998-2012. palisades, ny: Nasa socioeconomic data and applications center (sedac), http://dx.doi.org/10.7927/H4028PFS (2015).

[19] A. van Donkelaar, R. V. Martin, M. Brauer, B. L. Boys, Use of satellite observations for long-term exposure assessment of global concentrations of fine particulate matter, Environmental Health Perspectives 123 (2) (2015) $135-143$.

[20] W. S. Robinson, Ecological correlations and the behavior of individuals, American Sociological Review 15 (1950) 351-357.

[21] C. A. Gotway, L. J. Young, Combining incompatible spatial data, Journal of the American Statistical Association 97 (459) (2002) 632-648.

[22] C. Jackson, N. Best, S. Richardson, Hierarchical related regression for combining aggregate and individual data in studies of socio-economic disease risk factors, Journal of the Royal Statistical Society. Series A (Statistics in Society) 171 (2008) 159-178. 


\section{Appendix}

420 $\mathrm{R}$ code for combined analysis of point-level and area-level data using INLA and SPDE.

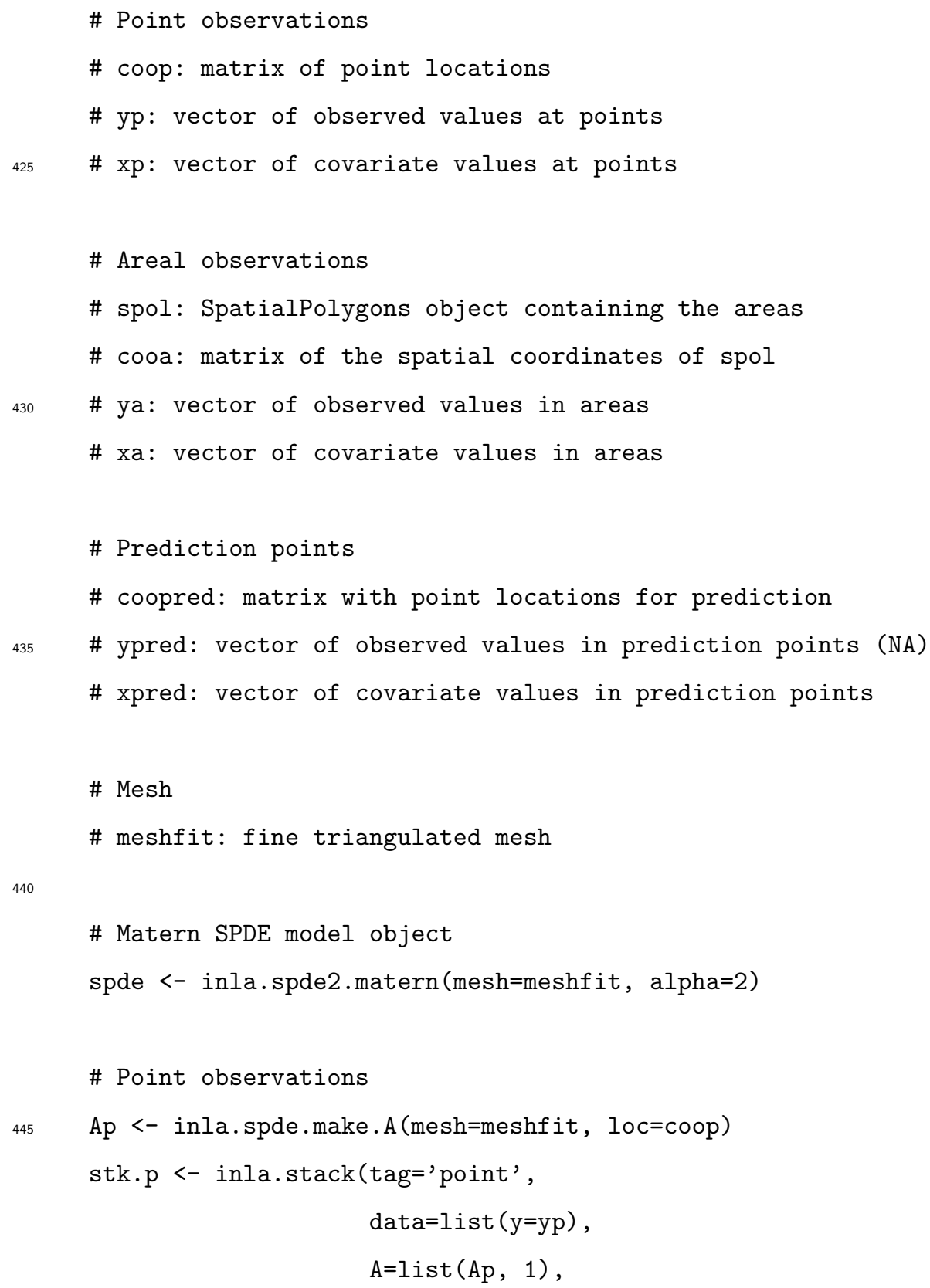


effects $=1$ ist $(s=1$ : spde $\$$ n. spde, data.frame $(b 0=1, x=x p)))$

450

\# Areal observations

locin <- meshfit\$loc [as.vector(which(!is.na(over(SpatialPoints(meshfit\$loc), spol)))), ]

block <- $\operatorname{rep}(0, \operatorname{nrow}($ locin $))$

for ( $i$ in $1:$ length $(\operatorname{spol}))\{$

455

block[as.vector(which(!is.na(over(SpatialPoints(locin), spol[i]))))]<- i

\}

Aa <- inla.spde.make.A(mesh=meshfit, loc=locin, block=block, block.rescale="sum") stk.a <- inla.stack(tag='areal',

data=list $(\mathrm{y}=\mathrm{ya})$,

460

$\mathrm{A}=\mathrm{list}(\mathrm{Aa}, 1)$,

effects=list $(s=1: \operatorname{spde} \$ n$.spde, data.frame $(b 0=1, x=x a)))$

\# Prediction points

Apred <- inla.spde.make.A (mesh=meshfit, loc=coopred)

465

stk.pred <- inla.stack (tag='pred',

data $=$ list ( $y=y p r e d)$,

$A=l$ ist (Apred, 1),

effects $=1$ ist $(s=1: \operatorname{spde} \$ n$. spde, data.frame $(b 0=1, x=x p r e d)))$

470

\# Stack

stk.full <- inla.stack(stk.p, stk.a, stk.pred)

\# Fit model

formula $<-y \sim 0+b 0+x+f(s$, model=spde $)$

475

res <- inla(formula, data=inla.stack.data(stk.full),

control.predictor=list (compute=TRUE, A=inla.stack.A(stk.full))) 
Parameters values

S1:

$\beta_{0}=0$

$\beta_{c}=-$

$\sigma^{2}=4$

$\rho=0.7$

S2:

$\beta_{0}=0$

$\beta_{c}=-$

$\sigma^{2}=1$

$\rho=0.1$
Simulated surfaces
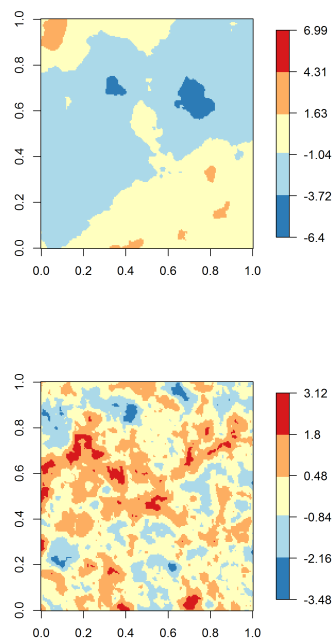

S3:

$\beta_{0}=0$

$\beta_{c}=2$

$\sigma^{2}=4$

$\rho=0.7$
Areal observations
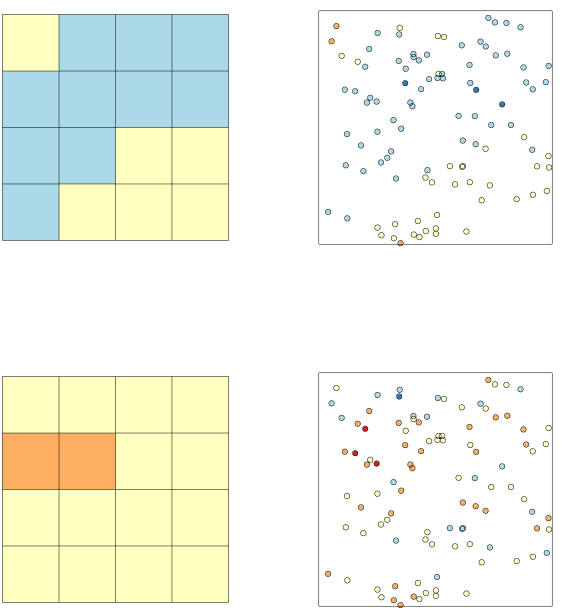

S4

$\beta_{0}=0$

$\beta_{c}=2$

$\sigma^{2}=1$

$\rho=0.1$
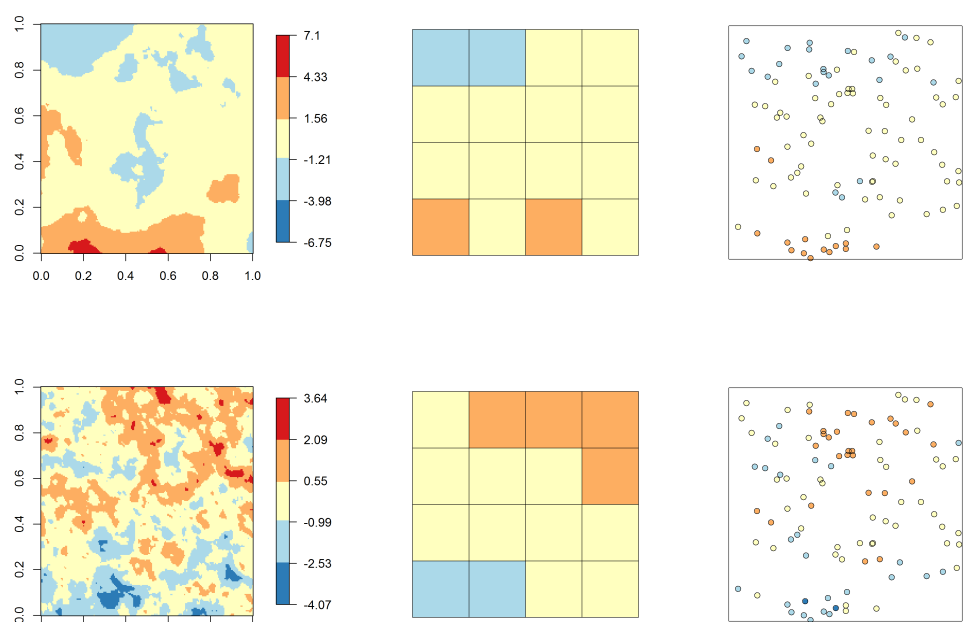

Figure 6: Simulated surfaces and point and areal observations used in the performance study. 


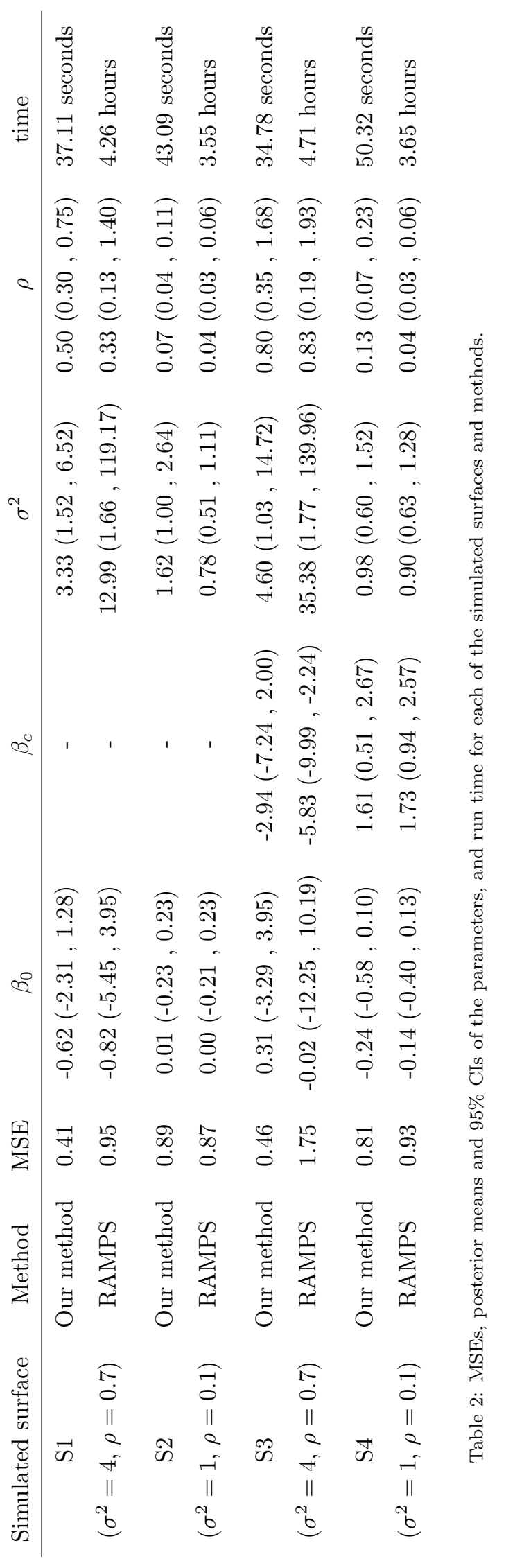



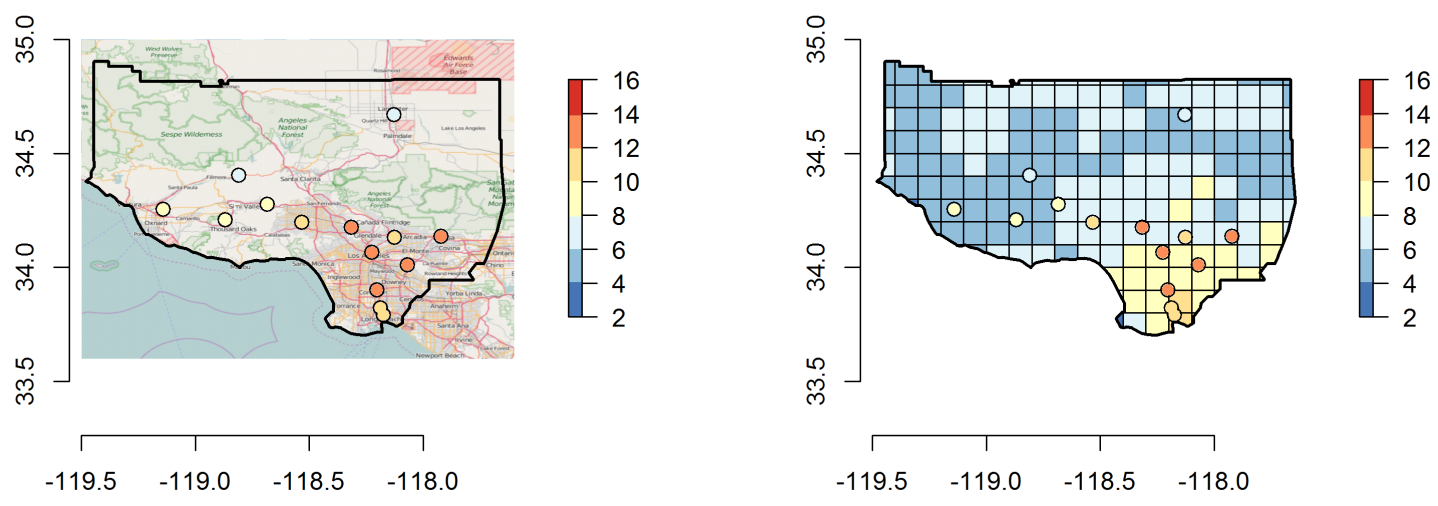

Figure 7: $\mathrm{PM}_{2.5}$ concentration (micrograms per cubic meter) in monitoring stations (left) and raster grid together with monitoring stations (right) in Los Angeles and Ventura counties in 2011.

\begin{tabular}{cccc} 
& $\beta_{0}$ & $\sigma^{2}$ & $\rho$ \\
\hline Joint analysis & $7.31(6.46,8.22)$ & $2.72(0.94,6.35)$ & $1.20(0.60,2.22)$ \\
Areal analysis & $7.50(7.09,7.91)$ & $3.74(1.65,7.50)$ & $0.85(0.52,1.34)$ \\
Point analysis & $9.51(5.42,12.94)$ & $7.00(1.78,20.2)$ & $0.74(0.31,1.55)$
\end{tabular}

Table 3: Posterior means and $95 \%$ CIs of the model parameters in the air pollution study. 
Mean
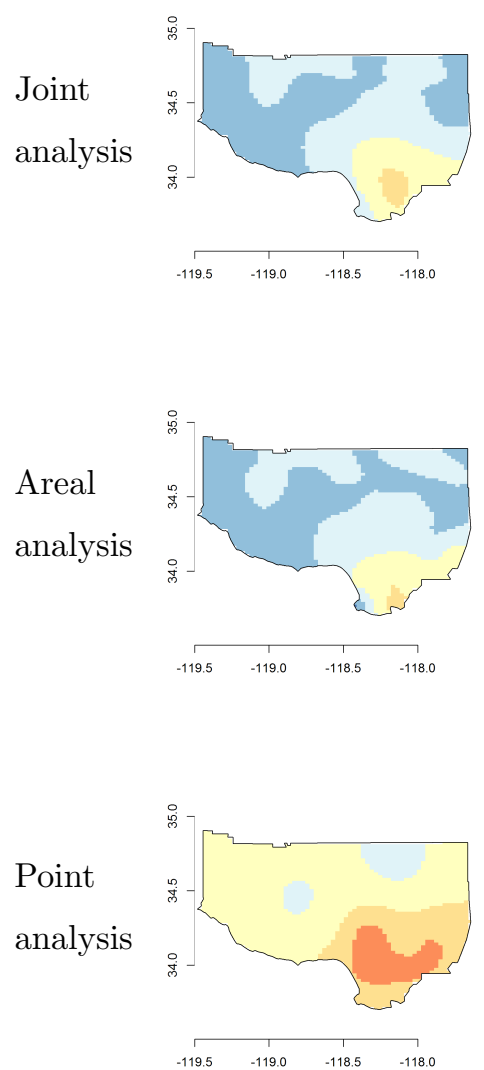

2.5 percentile
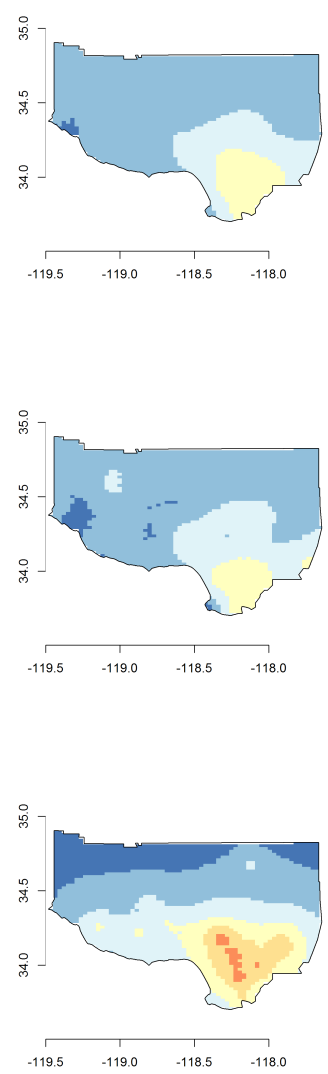

97.5 percentile
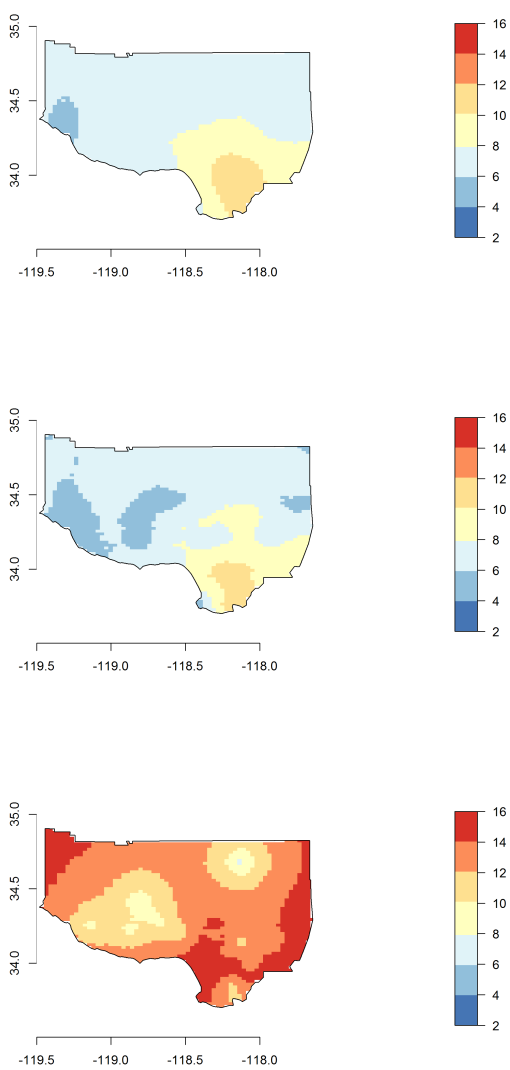

Figure 8: Posterior $\mathrm{PM}_{2.5}$ concentration (micrograms per cubic meter) by type of analysis, Los Angeles and Ventura counties, 2011. 